Formación de Recursos Humanos en el Hospital Odontológico Universitario de la FOUNLP

Teresa A. Butler

Revista ES (en y sobre Educación Superior)

Vol.1, N¹-2 / Fecha de publicación: 27/12/2021

e-ISSN: $2718-6539$

https://revistas.unlp.edu.ar/ES/index

IIES - Facultad de Odontología

DOI: https://doi.org/10.24215/27186539e007

\title{
Formación de Recursos Humanos en el Hospital Odontológico Universitario de la FOUNLP
}

\author{
Training of Human Resources at the Dental Hospital of the National \\ University of La Plata
}

\section{Formação de Recursos Humanos no Hospital Odontológico da Universidade Nacional de La Plata}

Doctora Teresa A. Butler

Secretaria de Articulación Docencia Asistencia FO - UNLP

dikybutler@yahoo.com.ar

\section{Resumen}

A partir de la realización de tres seminarios convocados por la Organización Panamericana de la Salud (OMS) 1962 y 1966, se produjeron cambios 
sustanciales en las diferentes escuelas y facultades de Odontología de América Latina, pensando en la formación de recursos humanos para la atención de la salud oral de la población. Fueron América Latina y el Caribe quienes inician las diferentes reformas de salud, teniendo en cuenta la equidad y la atención primaria de la salud (APS). Considerando los cambios socio-económicos, culturales y científicos de la actualidad, y dentro del marco de gratuidad, exclusivo, de excelencia y ético de la Universidad Nacional de La Plata, se formula este proyecto de intervención desde el Hospital Odontológico de la Facultad de Odontología de la UNLP. Dicho trabajo es aplicado al recurso humano que se forma en el Hospital Odontológico Universitario de la facultad de Odontología de la UNLP. El objetivo general es diseñar estrategias de formación en servicio de los alumnos de grado de la carrera de odontología de la UNLP, que realizan sus prácticas en el hospital escuela de la FOLP que contribuya a la formación continua e integral de los mismos. El proyecto será realizado en tres etapas: inicial, intermedia y final. Las mismas se delinearán a partir de un diagnóstico situacional, para posteriormente elaborar, organizar y planificar las diferentes estrategias a seguir, intentando reformar la formación de los recursos humanos con la contención de los pacientes asistidos en el mencionado hospital. Las diferentes acciones las irán desarrollando los diferentes actores involucrados. Entre las reflexiones más relevantes se dice que el modelo propuesto de intervención tendrá flexibilización y un plan de mejoras continuas, basado en el mejoramiento de la salud de la comunidad, y la minimización de las desigualdades.

\section{Abstract}


From the realization of three seminars by the Panamerican Health Organization (WHO) 1962 and 1966, substantian changes took place and the different schools and faculties of Dentistry in Latin America, thinking about the training of human resources for the attention of the oral health of the population. It was Latin America and the Caribbean that initiated the different health reforms taking into account equity and primary health care ( $\mathrm{PHC})$. Conssidering the current socio-economic, cultural and scientific changes, whithin the framework of free, exclusive, excellent and ethical at the National University of la Plata, this intervention project is formulated from the Dental Hospital of the faculty of Dentistry of the UNLP. This work is applied to the human resource that is formed at the University Dental Hospital of the UNLP School of Dentistry. The general aim is to desing in- service training strategies for undergraduate students of dental career at the UNLP, who carry out their internships at the FOLP School Hospital that contributes to their continuous and comprenhensive training. The projet will be carried out in three stages initial, intermediate and final.They will be delineated base on a situational diagnosis, to later elaborate, organized and plan the different strategies to follow, trying to reform the training of human resoucers with the cointainments of patients assisted in the aforementiond hospital. The different actions will be developed of differente actors involved. Among the most relevant reflections, it is said that the proposed intervention model will have flexibility and a plan for continuous improvement based on improving the health on the community minimizing inequalities.

\section{Resumo}

A partir da realização de três seminários convocados pela Organização PanAmericana da Saúde (OMS) em 1962 e 1966, mudanças substanciais 
ocorreram nas diferentes escolas e faculdades de Odontologia da América Latina, pensando na formação de recursos humanos para o cuidado da saúde bucal. saúde da população. Foi a América Latina e o Caribe que iniciaram as diferentes reformas da saúde, levando em consideração a equidade e a atenção primária à saúde (APS). Tendo em conta as actuais transformações socioeconómicas, culturais e científicas, e no âmbito do enquadramento gratuito, exclusivo, de excelência e ético da Universidade Nacional de La Plata, este projecto de intervenção é formulado a partir do Hospital de Odontologia da Faculdade de Odontologia da UNLP. Este trabalho é aplicado ao recurso humano formado no Hospital Odontológico Universitário da Faculdade de Odontologia da UNLP. O objetivo geral é traçar estratégias de formação em serviço para alunos de graduação em odontologia da UNLP que realizam estágio no hospital universitário da FOLP que contribuam para sua formação contínua e integral. O projeto será realizado em três etapas: inicial, intermediária e final. Serão traçados a partir de um diagnóstico situacional, para posteriormente elaborar, organizar e planejar as diferentes estratégias a seguir, buscando reformar a formação de recursos humanos com a contenção dos pacientes atendidos no referido hospital. As diferentes ações serão desenvolvidas pelos diferentes atores envolvidos. Entre as reflexões mais relevantes, afirma-se que o modelo de intervenção proposto terá flexibilidade e um plano de melhoria contínua, baseado na melhoria da saúde da comunidade e na minimização das desigualdades.

\section{Palabras clave}

Formación, recurso humano, Hospital Odontológico, asistencia, comunidad. 


\section{Keywords}

Training, human resource, Dental Hospital, assistance, community.

\section{Palavras chave}

Treinamento, recursos humanos, Hospital Odontológico, assistência, comunidade.

\section{Problemáticas}

Las acciones de este trabajo se implementan mediante una red en la que participan el Ministerio de Educación de la Nación, el Ministerio de Salud de la Provincia, autoridades de la Universidad, el grupo de Gestión de la Facultad de Odontología, docentes de la Facultad de Odontología de la Universidad Nacional de La Plata, alumnos de cuarto y quinto años de la carrera de Odontología, residentes de la carrera de la especialización en Odontología Social y comunitaria, no docentes e individuos de la comunidad. Dicho Proyecto se organiza desde la Secretaría de Articulación Docencia Asistencial de la Facultad de odontología de la Universidad nacional de La Plata (FOUNLP); articulado con el Decanato, Vicedecano, y las demás Secretarías de la Gestión de dicha Casa de Altos Estudios; considerando que la Universidad es una institución pública y gratuita de la Educación Superior, abierta e inclusiva para toda la sociedad. El desafío que nos pone este proyecto es recuperar saberes previos y experiencias personales referidos al derecho de la salud, hacia un modelo inclusivo, y de atención de óptima calidad para avanzar sobre las transformaciones de las prácticas de la salud en el Hospital Odontológico de la FOUNLP. En consecuencia, el proceso de enseñanza -servicio que realiza el recurso humano de la FOUNLP, es de 
contenido ético, cultural, social y científico, general y de carácter multidisciplinario, con sentido creativo e innovador, comprometido con las transformaciones, y adaptado en forma permanente a las nuevas tendencias para satisfacer las necesidades reales del entorno próximo y de la sociedad.

Este proyecto se basa en la formación de Recursos Humanos en Salud Bucal, pensando en su participación como el derecho que permite la construcción de un espacio de atención pública que facilita el ejercicio de la ciudadanía en pos del Estado. Por ende, el eje central del mismo se basa en la formación integral de los estudiantes de Odontología de grado y posgrado, que deben cumplir con las competencias de la currícula de la FOUNLP, generando un espacio de aprendizaje y capacitación en el servicio odontológico, en un ámbito que conste de una tecnología adecuada; con la finalidad de generar estrategias con valor social y ciudadano, sustentadas en la asistencia, prevención, rehabilitación y contención de los individuos de la comunidad en el ámbito universitario. En esta organización de actividades participan también las Prosecretarías de Seguridad, Higiene y Recursos Sustentables y de Bioseguridad, como así también la central de esterilización, área de admisión de pacientes, y los docentes de las asignaturas básicas y clínicas integrales, de especialidades de grado y posgrado, la clínica de alta complejidad de la FOUNLP, según lo requiera el caso, articulados con la Secretaría de Articulación DocenciaAsistencial de la mencionada Institución.

La problemática que surge es la necesidad de aumentar los espacios físicos, el personal docente, personal de Bioseguridad y personal de esterilización, como así también la incorporación de tecnología de alta generación, para lograr el incremento de la cantidad de pacientes asistidos en el rango horario de lunes a viernes de 8 a 23 horas, y sábados de 8 a 17 horas, que desarrolla actividades 
en el Hospital Odontológico Universitario. Por ello, sería importante que la Institución formadora de estos recursos humanos pudiera contar con un presupuesto mayor.

El objetivo general de este trabajo es diseñar estrategias de formación en servicio de los alumnos de grado y Posgrado de la carrera de Odontología de la UNLP, que realizan sus prácticas en el Hospital Odontológico Universitario de la FOUNLP, contribuyendo en la formación continua e integral de los mismos.

Para poder alcanzar los objetivos propuestos se considerarán las estrategias educacionales, organizacionales y de comunicación; así como los factores políticos, socio-económicos, culturales, ambientales y conductuales.

El trabajo se desarrollará en diferentes etapas:

Inicial: en la cual se llevará a cabo el diagnóstico de situación y la evaluación inicial con la intervención de los representantes del Co-gobierno de la Universidad y de la FOLP.

Intermedia: que se llevará a cabo en el campo de acción (Hospital Odontológico Universitario de la FOUNLP). Para ello, se planificó un trabajo de Intervención, con la participación de todos los actores y un monitoreo permanente.

Final: se llevará a cabo la evaluación de los resultados enunciando las reflexiones.

Las estrategias utilizadas serán

a-Comunicación con las autoridades de la Facultad de Odontología de la Universidad Nacional de La Plata: se realizarán reuniones con el Decano, Vicedecana; Prosecretarios de Articulación Docencia Asistencial y Directores de dicha área de la FOLP_UNLP). Una vez analizado el diagnóstico epidemiológico y económico, y establecidos los problemas a resolver que 
afecta la formación de los recursos humanos en el Hospital Odontológico Universitario de la FOUNLP, se organizará la programación.

Para ello, se considerarán las siguientes competencias del hospital Odontológico Universitario de la FOLP_UNLP a considerar:

- El servicio de admisión y documentación clínica, que integra los movimientos y todos los servicios asistenciales que los alumnos de las diferentes asignaturas asistirán en sus prácticas, y en caso de ser necesario, en las áreas de atención especializada.

- La gestión operativa de los procesos administrativos de acceso y circulación asistencial de los pacientes.

- La organización y evaluación del proceso asistencial con el fin de mantener la cohesión entre la organización del alumno-paciente, para favorecer una utilización adecuada y eficiente de los recursos.

- La obtención de datos que permitan disponer al hospital universitario de información sobre la demanda existente y la actividad asistencial realizada.

b-Análisis de datos: se llevará a cabo mediante la recolección de datos cuali y cuantitativos, a través de la observación de las historias clínicas de los pacientes atendidos en forma diaria, por el período de un año, observando cuántos han culminado sus tratamientos orales. Se completará este análisis con entrevistas a docentes de las asignaturas clínicas de la FOUNLP, describiendo cuales son las fortalezas y debilidades en relación a los recursos humanos del hospital Odontológico Universitario de la FOLP, relacionados con la atención odontológica de los individuos de la comunidad. Se dictarán cursos de actualización de todas las especialidades de Odontología, sobre informática y sobre el uso y planificación de las diferentes tecnologías incorporada, sobre 
primeros auxilios dirigidos al personal docente, alumnos y personal no docente de la FOLP.

c) Seguimiento y actualización permanente de la atención de los pacientes que asisten al Hospital Odontológico Universitario de la FOUNLP.

Las diferentes acciones de la Secretaría se encuentran dentro de lo reglamentado por el Ministerio de Salud Pública de la Nación. Las Normas de Bioseguridad, reglamento de esterilización y validación de la calidad de la esterilización, se enmarcan dentro de la Resol. 1547/2007, del Ministerio de Salud Pública de la Nación.

\section{Análisis situacional}

El plan estratégico de la Formación de Recursos Humanos fue variando en diferentes décadas, de acuerdo a las necesidades del alumno y de la sociedad. En sus comienzos, el estudiante de odontología realizaba sus prácticas odontológicas durante los cinco años de la carrera en las clínicas destinadas para tales fines. Con el correr del tiempo, se crea el Hospital Escuela de la FOLP, donde se desarrollaban prácticas complementarias supervisadas por docentes de la facultad de Odontología de la UNLP, y urgencias, además de realizarse trabajos específicos. Posteriormente dicho hospital se convierte en el Hospital Odontológico de la Facultad de odontología de la UNLP actual, en el que se prestan servicios odontológicos, y desarrollan sus prácticas alumnos de la Especialización de la carrera de Odontología Social y Comunitaria, los residentes, y se practican atenciones de Posgrado como Implantología Oral, Cirugías, etc; asistiendo de esta forma a todos los integrantes de la comunidad que requieran servicios odontológicos. 
La formación del Recurso Humano de la FOUNLP, se realiza durante toda la carrera mediante observaciones y prácticas a través de la asignatura Odontología Preventiva y Social (OPS), que es el eje fundamental. Por lo tanto, la formación del Recurso Humano, siempre relacionada con las necesidades de la comunidad para definir los objetivos y el plan de la currícula. Ello responde a un diagnóstico de situación epidemiológico y económico de los recursos existentes, que serán expuestos a continuación.

\section{Situación actual}

La FOUNLP, a través de su relación directa con la Universidad Nacional de la Plata, y sus actores de cogobierno, son los encargados de establecer el protocolo del funcionamiento del Hospital Odontológico Universitario de la FOUNLP, para la asistencia odontológica a la comunidad, a través de las prácticas asistenciales que desarrolla el Recurso Humano en su formación profesional.

El financiamiento de dicho servicio está relacionado con los recursos financieros de autogestión, y presupuestos otorgados de la Universidad Nacional de La Plata, para la compra de insumos de alta tecnología, instrumental y aparatología específica.

La articulación está referida directamente a la formación del recurso Humano en Odontología, donde confluye la formación de los nuevos conocimientos con relevancia científica, que serán aplicados en los espacios de extensión, y por ende, con su aplicación en el servicio docencia que los alumnos llevan a cabo a través de sus prácticas hospitalarias en la Facultad de Odontología de la UNLP, sobre los individuos de la comunidad. 
La importancia de la formación de Recursos Humanos en Odontología como etapa de la formación académica, demanda el incremento del presupuesto anual para el desarrollo de las prácticas asistenciales dirigidas a la comunidad. De esta manera, se podría garantizar el desarrollo y seguimiento de los tratamientos integrales de los individuos de la comunidad. Los profesores pertenecientes a las asignatura clínicas que colaboran con la formación académica, e investigativa de los recursos humanos de la FOLP, sustentarán las acciones asistenciales mediante el seguimiento continuo de la realización y finalización de los tratamientos integrales de los pacientes que concurren diariamente al Hospital Escuela Universitario de la FOLP. El mismo se realizará mediante el sistema SIU hospitalario que se implementará en la FOLP.

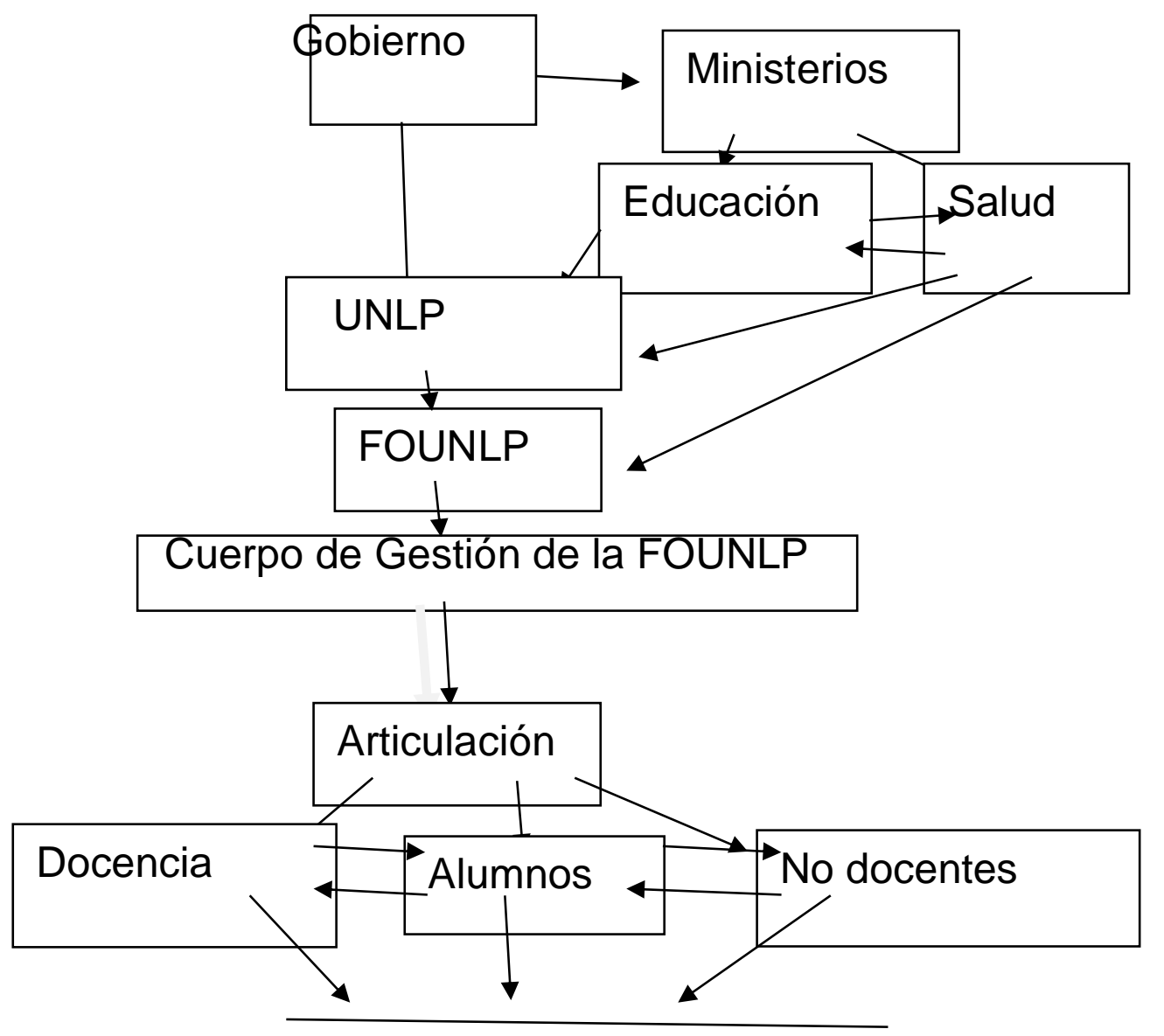




\section{Servicio a la comunidad}

\section{Reflexiones}

La formación del recurso humano en la FOUNLP insume gran parte del financiamiento que dispone dicha casa de Altos estudios, por ende, es necesario que en ámbito de la Gestión y Desarrollo, se incluyan alianzas entre diferentes instituciones para lograr el perfeccionamiento profesional y técnico que asegure la salud pública. Para que la misma sea relevante y produzca un impacto social, se deberá contar con una buena planificación, organización y política de salud.

Los hospitales públicos son parte del sistema de salud, teniendo un espacio muy importante dentro de su gobierno y gestión. Por un lado, los caracteriza el desafío de las políticas aplicadas en ellos, por el otro, la gestión de la parte administrativa y económica, necesaria para la asistencia a la comunidad. Para la autogestión es fundamental que posean recursos humanos idóneos en las acciones que desarrollan, que será transferido a la eficiencia sanitaria, incluyendo el servicio de las prestaciones dirigidas a los usuarios.

El modelo propuesto tendrá flexibilización y un plan de mejoras continuas.

La propuesta de este proyecto se basa en mejorar la salud de la comunidad y disminuir las desigualdades. Para ello es necesario observar las necesidades y cambios de la sociedad, y los desafíos que se deberán enfrentar, para lograr una atención odontológica de excelencia sin exclusión. 
Se observarán las respuestas obtenidas de todos los actores participantes (Directivos de la FOLP, alumnos, profesores, auxiliares de la salud, individuos de la comunidad, etc).

Se solicitará respaldo político (autoridades sanitarias y de gobierno) para aumentar los presupuestos para el mantenimiento de las prácticas asistenciales diarias.

Se mantendrán vínculos cercanos con otras entidades de la salud bucal, laboratorios, comunidades, universidades, Ministerios de salud y gobierno, para adecuar la intervención a los valores y necesidades de la comunidad.

Se considera importante articular con la Secretaría de Salud de la UNLP, Secretaría de Políticas Universitarias de la UNLP y con la Secretaría de Extensión de la FOLP, la continuación de la asistencia de odontológica en sectores de la comunidad vulnerables de la ciudad de La Plata y alrededores, mediante el recurso humano formado en la FOUNLP.

Es la pretensión de este proyecto que establezca una verdadera red comunicacional, entre el sistema universitario público, la salud pública de la zona, y la comunidad. Será un verdadero desafío institucional, cuyo eje de acción es la formación del recurso humano que transita por el Hospital odontológico Universitario de la FOUNLP, con el máximo nivel de jerarquía profesional y sentido ético-comunitario, involucrando a los actores directos y a otros indirectos tal como el Ministerio de Salud de la provincia, la Superintendencia de salud de la Nación, a los Municipios que tienen convenio con la FOUNLP a través de la carrera de Especialización en Odontología Social y Comunitaria (San Martín, Santa Teresita, Magdalena, etc) y al Gobierno Nacional a través del Ministerio de Educación de la Nación y la Secretaría de Políticas Universitarias. 


\section{Bibliografía}

- Tauber Fernando. Pensar la Universidad. Proyecto Institucional de la UNLP 2018-2022. $1^{\circ}$ edición, abril de 2018.

- Tauber Fernando. Tesis Doctoral: La comunicación en la planificación y gestión para el desarrollo de las Instituciones universitarias públicas argentinas: el caso de la Universidad de La Plata en el trienio junio 2004mayo 2007.

- Yepez, Patricio. El Proceso de Organización de Facultades, Escuelas y Departamentos de Odontología (OFEDO/UDUAL). 2018

- Memorias. Encuentro Universidad Latinoamericana y Salud de la Población. Retos y desafíos para el siglo XXI. 16-19 noviembre, La Habana, Cuba.

- Shortell SM. Continuity of medical care: conceptualization and measures . Medical Care 1976; 14:337-91

- Huertas Franco. El método PES. Entrevista a Carlos Matus. Edit. Acladir

- Botero, Ángela, Restrepo Horacio y colab. Educación médica y salud. Enero, febrero-marzo; 1971; vol.5, N|1.

- Odontología- Universidad- Sociedad. Rev. Salud Pública Parag. 2013, vol.3; N 2; julio-diciembre 2013

- Godoy Ortiz, Aníbal. Los cambios en la sociedad. Rev. Soc.Chilena, 2016

- Proenza Díaz, Tamara y colab. Caracterización de la formación de Doctores en la Universidad de la Habana. Pedagogía Universitaria, Vol. $X X, N 3,2015$ 
- Martín Aiello y Norberto Fernandez Lamarra. Políticas y gestión en la Universidad. Revista Argentina de Educación Superior Conocimiento y Difusión. 2017

- XXI Jornadas de Economía Solidaria de Aragón (REAS). Del 6 al 9 de junio de 2016.

- Burton R Clark. El sistema de Educación Superior. Edit. Nueva Imagen. Universidad Autónoma Metropolitana, México; 1991.

- Jan Servaes: Investigación participativa en el área de la Comunicación. 2004.

- Argiris Chris. Aprendiendo a aprender para el desarrollo. 2018

- Tomas Leandro Juan, Medina María Mercedes; Albarracín Silvia; Rueda Leticia; Coscarelli Nélida; y colab. Experiencia de aprendizaje servicio en la FOLP. (2008)

- López G.J.B. Estrategias curriculares alternativas en la enseñanza. V Conferencia de OFEDO/UDUAL. Cuenca, Ecuador, 1989.

- Foucault, M. El nacimiento de la clínica. Siglo XXI, México, 1966.

- Cámara López, Víctor. Contexto de la Educación Odontológica. Educ. Med Salud, vol 22 (2), 1988.

- -Albarracín, Silvia; Coscarelli Nelly. Atención Primaria en SALUD (APS). Congreso de Políticas Sociales. Corrientes. Octubre 2008

- Giordano, Carlos José. Tesis de Doctorado: 23 Tesis sobre la Tesis. La comunicación para la transformación de la gestión educativa. Junio 2009.

- Ley 24.521 de Educación Superior, del 07/08/90

- Doberti, Ignacio. Los aspectos distintivos de la universidad.

- Carlos Matus. Política, Planificación y Gobierno. 1987. 
- Payares E; Arango A. Consideraciones socio-históricas de la Odontologia en Colombia y Antioquía- Siglo XX- Medellín. Promotora de ediciones y Comunicaciones SA

- Caballé, Marcelo: Problemática de la transferencia en Universidades públicas: El caso de la Universidad Nacional de La Plata. 